UNIVERSIDADE DE SÃO PAULO

ESCOLA DE EDUCAÇÃO FÍSICA E ESPORTE

\title{
EFEITOS DA INFORMAÇÃO VERBAL NO ACOPLAMENTO ENTRE A INFORM AÇÃO VISUAL E OSCILAÇÃO CORPORAL
}

Alaércio Perotti J únior

SÃO PAULO

2006 


\title{
EFEITOS DA INFORMAÇÃO VERBAL NO ACOPLAMENTO ENTRE A INFORM AÇÃO VISUAL E OSCILAÇÃO CORPORAL
}

\author{
ALAÉRCIO PEROTTI JÚNIOR
}

Tese apresentada à Escola de E ducação Física e Esporte da Universidade de São Paulo, como requisito parcial para obtenção do grau de Doutor em E ducação Física.

ORIENTADOR: PROF. DR. GO TANI CO-ORIENTADOR: PROF. DR. J OSÉ ANGELO BARELA 


\section{AGRADECIMENTOS}

Ao Dr. Go Tani, pela orientação, confiança, amizade, paciência e pelos ensinamentos transmitidos durante todo o processo do doutorado.

Ao Dr. José Angelo Barela, pela orientação, pelos ensinamentos, confiança, amizade, fundamentais para a realização e conclusão deste trabalho.

Ao Dr. Umberto César Corrêa, um grande amigo, pelo incentivo, sugestões, considerações e ajuda, em todos os momentos, para este trabalho.

Ao Dr. Ricardo Demétrio de Souza Petersen pela contribuição a este trabalho.

À Dra Suely Santos pela colaboração e sugestões.

Ao Dr. Sérgio Tosi Rodrigues pelas sugestões e críticas a este trabalho.

À MS Paula Polastri, em especial, pela amizade, compreensão, auxílio em todos os momentos e contribuições para este trabalho.

Aos amigos do LACOM, Andréa, Cássia, Cássio, Herbert, João, Jorge, Luciano, Luis Dantas, Piraju, Rejane, Roberto, Welber, pelas críticas e sugestões a este trabalho.

Ao Matheus e a Carol, em especial, pelo auxílio e por estarem sempre disponíveis durante todo o processo de coleta de dados.

Aos amigos do Laboratório para Estudo do Movimento (UNESP-Rio Claro) sempre dispostos a ajudar, em especial Dani, Mário, Ivan, Thátia, Paulão, Priscila, Ana Paula, Maria Solange.

A todos os voluntários que participaram deste estudo. 
Aos funcionários da Secretaria de Pós-Graduação, em especial, Maria de Lourdes e Ilza.

Ao Gabriel e Reginaldo, pelo auxilio e dicas de informática.

À Giselda, pela amizade, auxilio e contribuição para este trabalho.

Aos meus alunos, pela participação e ajuda, em especial ao Thiago, Maicon, Philipe, Everton, Adriano e Fábio.

À Neuci, minha esposa e companheira, e a minha filha, Thais, pela compreensão e paciência.

À minha mãe Elza e minha irmã Cibele, pela ajuda nos momentos que necessitei estar ausente.

Aos amigos Beto, Jaime, Julia, Sr. Osmar Bacan, Adimir Nogueira e João Ferraz pela colaboração.

Dedico este trabalho ao meu pai, Alaércio Perotti, pelo exemplo de vida, integridade, apoio e incentivo às minhas escolhas (In Memorian). 


\section{SUM ÁRIO}

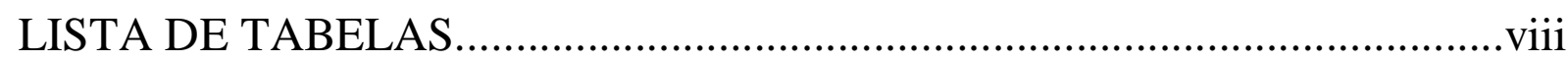

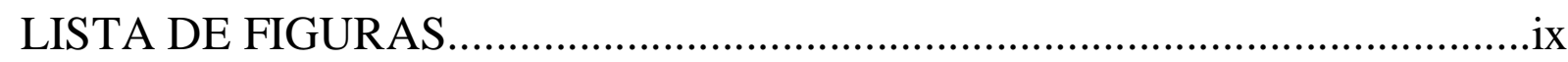

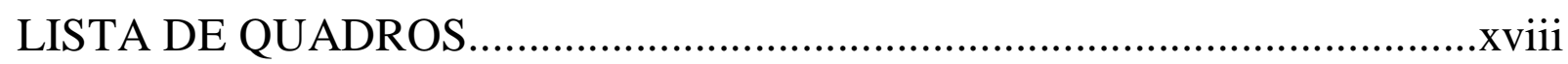

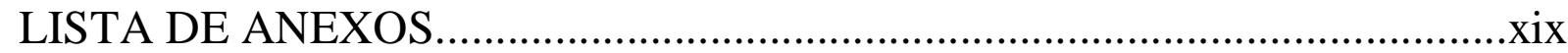

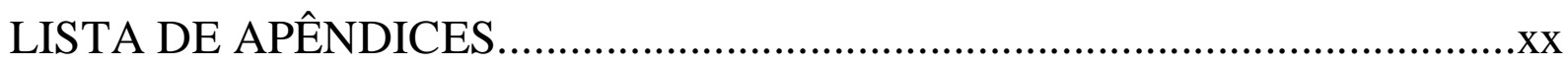

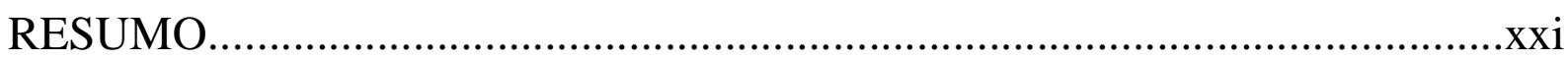

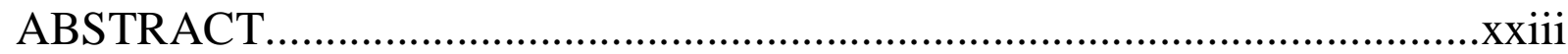

1 INTRODUÇÃO

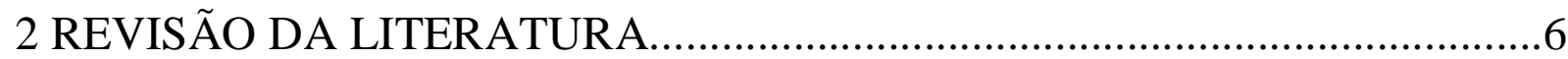

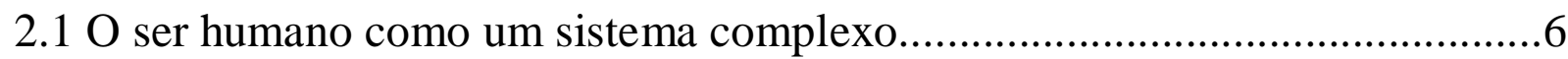

2.2 Uma breve revisão histórica dos estudos de desenvolvimento motor.............10

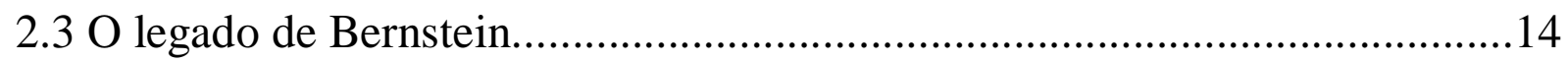

2.4 O legado de Gibson.................................................................................16

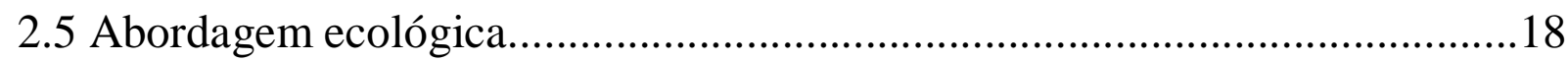

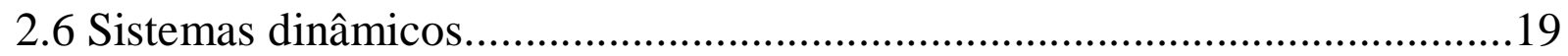

2.7 O papel das restrições..................................................................................23

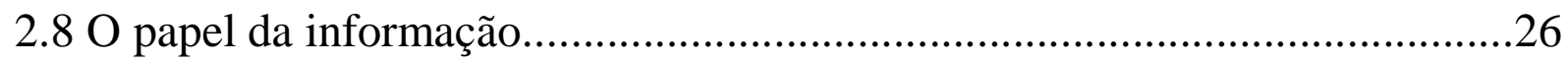

2.9 Dinâmica intrínseca e informação comportamental......................................29

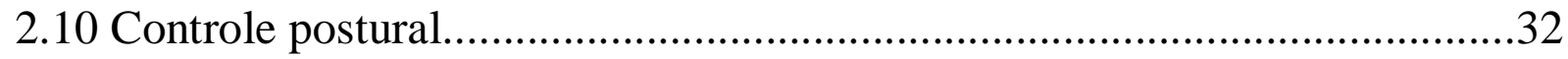

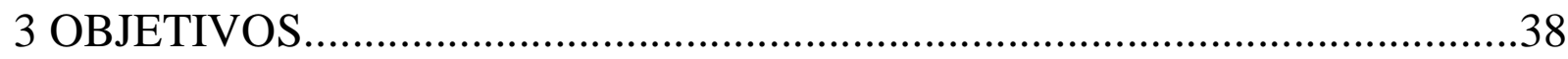

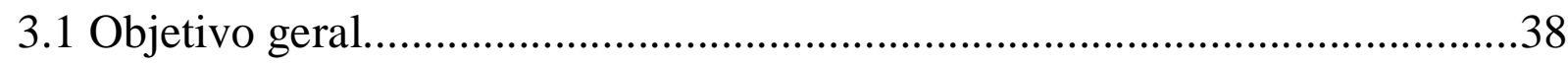




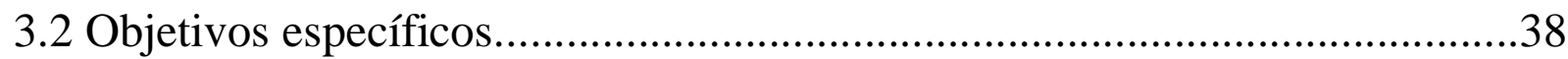

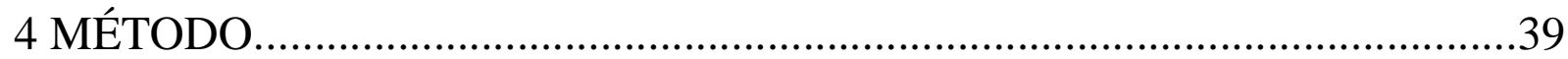

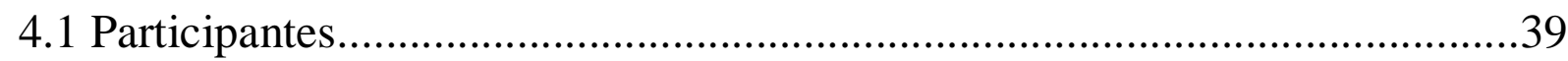

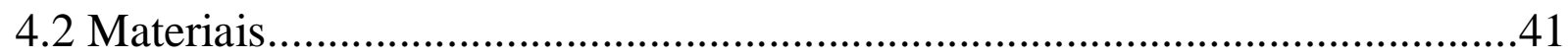

4.3 Experimento 1 - Com e sem movimento discreto da sala móvel..................45

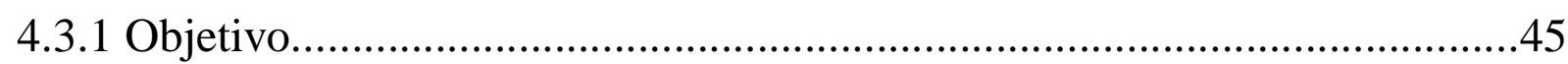

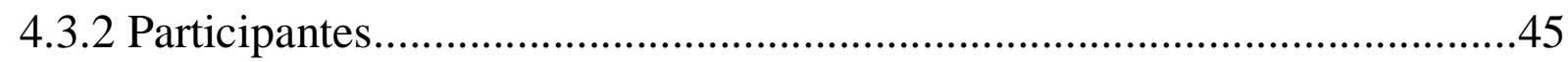

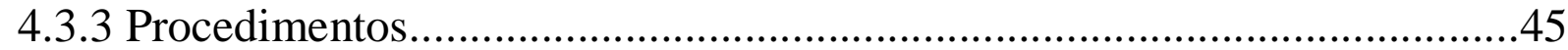

4.3.4 Decodificação e tratamento dos dados........................................................48

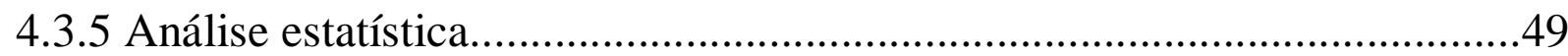

4.4 Experimento 2 - Com movimento contínuo da sala móvel............................50

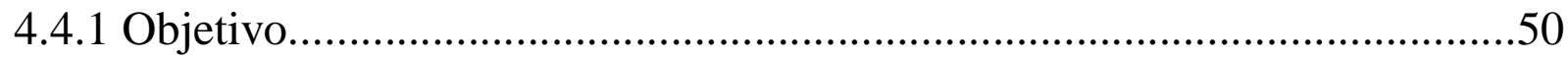

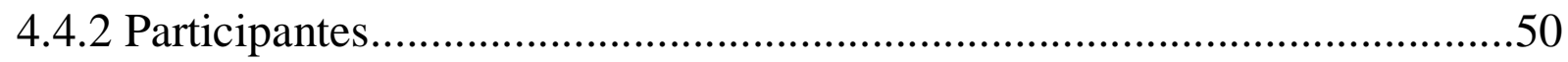

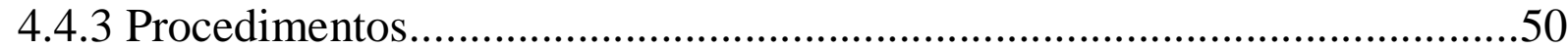

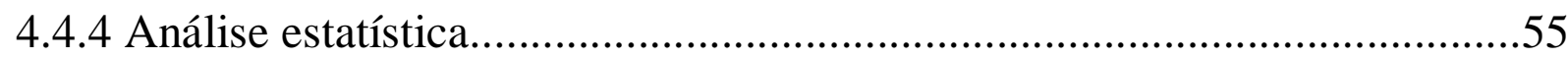

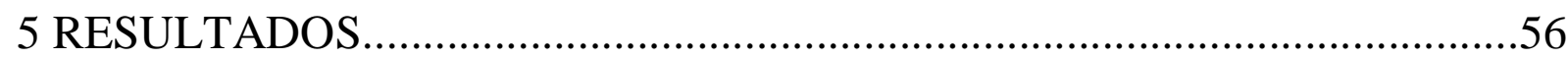

5.1 Movimentação discreta da sala móvel...........................................................56

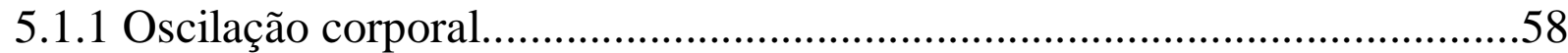

5.1.2 Relacionamento entre informação visual, fornecimento de informação verbal e oscilação corporal........................................................................63

5.1.3 Deslocamento corporal e tempo de reversão.................................................66

5.2 Condição experimental com movimentação contínua da sala móvel.............70 
5.2.1 Oscilação corporal com e sem movimento da sala móvel e sem o fornecimento de qualquer informação verbal...........................................70

5.2.2 Amplitude e freqüência média de oscilação...............................................75

5.3 Acoplamento entre informação visual e oscilação corporal - coerência, ganho, fase relativa e desvio angular.........................................................

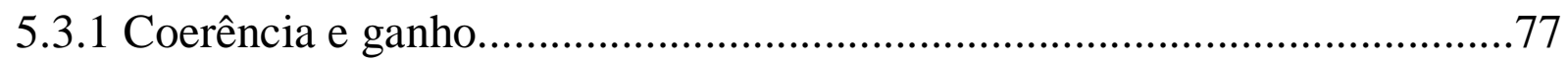

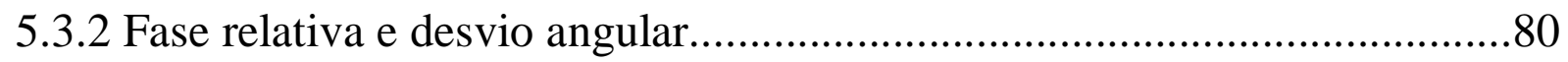

5.4 Oscilação corporal com e sem movimento da sala móvel com o fornecimento

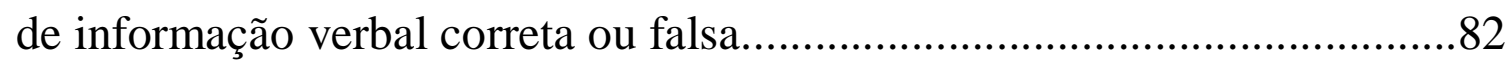

5.5 Comportamento dos participantes frente a movimentação ou não da sala móvel e ao fornecimento de informação verbal correta e falsa na ação de

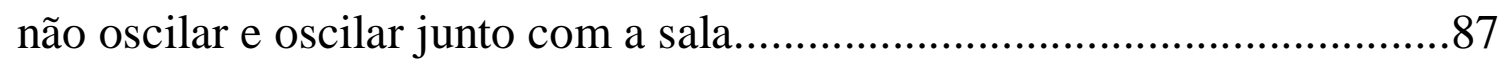

5.5.1 Amplitude e freqüência média de oscilação..............................................87

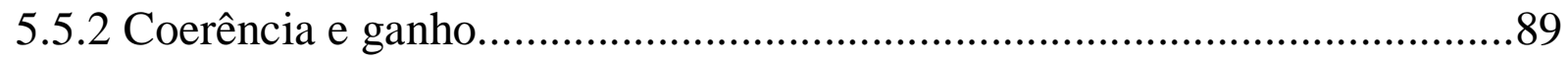

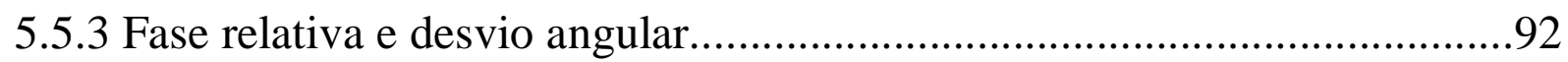

6 DISCUSSÃO

6.1 Movimento discreto da sala móvel.............................................................97

6.2 Oscilação corporal com e sem movimento da sala e sem fornecimento de informação verbal e solicitação da ação....................................................99

6.3 Oscilação corporal com o movimento da sala e sem informação verbal a respeito da movimentação da sala e solicitação de ação.............................100

6.4 Oscilação corporal com e sem movimento da sala móvel com o fornecimento de informação verbal e solicitação de uma ação...........................................102 
6.5 Controle postural frente ao fornecimento de informações verbais e das manipulações sensoriais. 107

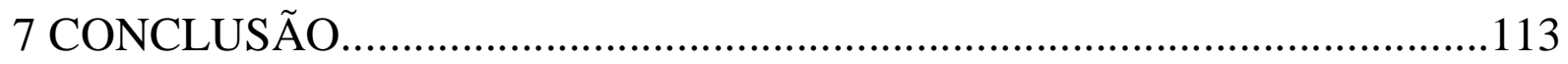

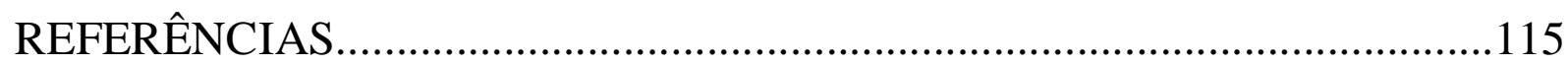

ANEXO I - Carta do comitê de ética...............................................................133

ANEXO II - Termo de consentimento........................................................134

APÊNDICE I - Planilha contendo os dados do experimento 1 - Com e sem movimento discreto da sala móvel..........................................136

APÊNDICE II - Planilha contendo os dados do experimento 2 - Com e sem movimento contínuo da sala móvel.......................................142 


\section{LISTA DE TABELAS}

TABELA 1 - Médias e desvio padrão da idade (em meses) dos participantes dos dois grupos etários nos dois experimentos. 


\section{LISTA DE FIGURAS}

FIGURA 1 - Informação versus entropia (MILLER, 1978). 9

FIGURA 2 - Esquema das três fontes gerais de restrições para ação motora (NEWELL, 1986).

FIGURA 3 - Várias possibilidades para uma mensagem buscar um atrator (HAKEN, 2000). .28

FIGURA 4 - Um outro exemplo de como as mensagens podem buscar um novo atrator (HAKEN, 2000). 29

FIGURA 5 - Vista frontal da sala móvel a ser utilizada no experimento. .42

FIGURA 6 - Servo-motor e cilindro de um eixo acoplados à estrutura da sala móvel. .42

FIGURA 7 - Vista lateral da unidade de sensores do sistema OPTOTRAK. .....43 FIGURA 8 - Vista frontal do local, com os equipamentos que foram utilizados no estudo.

FIGURA 9 - Exemplo da oscilação corporal na direção ântero-posterior de uma criança em uma tentativa em que a sala foi movimentada de forma discreta se aproximando e com o fornecimento de informação correta na ação solicitada para não oscilar, permanecendo o mais estático possível. 
FIGURA 10 - Exemplo da oscilação corporal na direção ântero-posterior de um adulto jovem em uma tentativa em que a sala foi movimentada de forma discreta se afastando e com o fornecimento de informação falsa na ação solicitada para não oscilar, permanecendo o mais estático possível. .58

FIGURA 11 - Amplitude média de oscilação na direção ântero-posterior do grupo de crianças e do grupo de adultos jovens na manutenção da postura ereta antes e após a movimentação discreta da sala, com o fornecimento de informação correta na ação solicitada para não oscilar, permanecendo o mais estático possível (painel a) e com o fornecimento de informação falsa (painel b). .60

FIGURA 12 - Amplitude média de oscilação na direção ântero-posterior do grupo de crianças e do grupo de adultos jovens na manutenção da postura ereta antes e após a movimentação discreta da sala aproximando e afastando, com o fornecimento de informação correta na ação solicitada para oscilar, junto com a sala (painel a) e com o fornecimento de informação falsa (painel b). 61

FIGURA 13 - Amplitude média de oscilação na direção ântero-posterior do grupo de crianças e do grupo de adultos jovens na manutenção da postura ereta antes e após a movimentação discreta da sala aproximando e afastando, com o fornecimento de informação correta na ação solicitada para oscilar, em sentido oposto ao movimento da sala (painel a) e com o fornecimento de informação falsa (painel b). 
FIGURA 14 - Coeficiente de correlação entre a movimentação da sala e a oscilação corporal do grupo de crianças e do grupo de adultos jovens na movimentação discreta da sala aproximando e afastando, com o fornecimento de informação correta (a,c,e) e falsa $(b, d, f)$ durante a manutenção da postura ereta na ação de não oscilar, oscilar junto e oscilar em sentido oposto. .64

FIGURA 15 - Diferença temporal entre a movimentação da sala e a oscilação corporal do grupo de crianças e do grupo de adultos jovens na movimentação discreta da sala aproximando e afastando, com o fornecimento de informação correta $(a, c, e)$ e falsa $(b, d, f)$ durante a manutenção da postura ereta na ação de não oscilar, oscilar junto e oscilar em sentido oposto.

FIGURA 16 - Deslocamento corporal na direção ântero-posterior do grupo de crianças e do grupo de adultos jovens na movimentação discreta da sala aproximando e afastando, com o fornecimento de informação correta (a, c, e) e falsa (b, d, f) durante a manutenção da postura ereta na ação de não oscilar, oscilar junto e oscilar em sentido oposto.

FIGURA 17 - Tempo de reversão na direção ântero-posterior do grupo de crianças e do grupo de adultos jovens na movimentação discreta da sala aproximando e afastando, com o fornecimento de informação correta (a, c, e) e falsa (b, d, f) durante a manutenção da postura ereta na ação de não oscilar, oscilar junto e oscilar em sentido oposto. 
FIGURA 18 - Exemplo de séries temporais da oscilação corporal nas direções ântero-posterior (painéis a e b) e médio-lateral (painéis c e d) de uma criança de 8 anos (painéis a e c) e de um adulto jovem (painéis $b$ e d) em uma tentativa em que a sala não foi movimentada. 70

FIGURA 19 - Exemplos de séries temporais de uma criança de 8 anos ao longo de uma tentativa mostrando o movimento da sala e a oscilação corporal na direção ântero-posterior (painel a), a fase relativa entre o movimento da sala e a oscilação corporal (painel b) e a análise espectral tanto do movimento da sala quanto da oscilação corporal (painel c) em uma tentativa em que a sala foi movimentada na freqüência de $0,2 \mathrm{~Hz}$ e o participante permaneceu a uma distância de $100 \mathrm{~cm}$. Nota: nos painéis a e b, a linha escura se refere ao movimento da sala e a linha mais clara às oscilações corporais dos participantes. 
FIGURA 20 - Exemplos de séries temporais de um jovem adulto ao longo de uma tentativa mostrando o movimento da sala e a oscilação corporal na direção ântero-posterior (painel a), a fase relativa entre o movimento da sala e a oscilação corporal (painel b), e a análise espectral tanto do movimento da sala quanto da oscilação corporal (painel c), em uma tentativa em que a sala foi movimentada na frequiência de $0,2 \mathrm{~Hz}$ e o participante permaneceu a uma distância de $100 \mathrm{~cm}$. Nota: nos painéis a e c, a linha escura se refere ao movimento da sala e a linha mais clara às oscilações corporais dos participantes. .72

FIGURA 21 - Exemplos de séries temporais de um jovem adulto ao longo de uma tentativa com a inclusão dos dados da movimentação da sala em uma tentativa em que a sala não foi movimentada mostrando o movimento da sala e a oscilação corporal na direção ânteroposterior (painel a), a fase relativa entre o movimento da sala e a oscilação corporal (painel b), e a análise espectral tanto do movimento da sala quanto da oscilação corporal (painel c) em uma tentativa em que a sala foi movimentada na frequiência de $0,2 \mathrm{~Hz}$ e o participante permaneceu a uma distância de $100 \mathrm{~cm}$. Nota: nos painéis a e c, a linha escura se refere ao movimento da sala e a linha clara às oscilações corporais dos participantes. 
FIGURA 22 - Médias e desvios padrão da amplitude média de oscilação nas condições sala com movimento e sala sem movimento, para os dois grupos etários sem o fornecimento de informação. .76

FIGURA 23 - Médias e desvios padrão da frequiência média de oscilação nas condições sala com movimento e sala sem movimento, para os dois grupos etários sem o fornecimento de informação. .76

FIGURA 24 - Médias e desvios padrão da coerência, nas duas condições em que a sala foi movimentada e a sala não foi movimentada, para os dois grupos etários. .78

FIGURA 25 - Médias e desvios padrão da coerência, nas duas condições em que a sala foi movimentada e a sala não foi movimentada, para os dois grupos etários.

FIGURA 26 - Médias e desvios padrão do desvio angular, nas duas condições em que a sala foi movimentada e a sala não foi movimentada, para os dois grupos etários.

FIGURA 27 - Médias e desvios padrão da fase relativa, nas duas condições em que a sala foi movimentada e a sala não foi movimentada, para os dois grupos etários. .81 
FIGURA 28 - Exemplos de séries temporais de uma criança de 8 anos ao longo de uma tentativa mostrando o movimento da sala e a oscilação corporal na direção antero-posterior (painel a), a fase relativa entre o movimento da sala e a oscilação corporal (painel b), e a análise espectral tanto do movimento da sala quanto da oscilação corporal (painel c), em uma tentativa em que foi fornecida uma informação correta e o participante foi solicitado a não oscilar. Nota: nos painéis a e c, a linha escura se refere ao movimento da sala e a linha mais clara às oscilações corporais dos participantes.

FIGURA 29 - Exemplos de séries temporais de uma criança de 8 anos ao longo de uma tentativa mostrando o movimento da sala e a oscilação corporal na direção antero-posterior (painel a), a fase relativa entre o movimento da sala e a oscilação corporal (painel b), e a análise espectral do movimento da sala quanto da oscilação corporal (painel c), em uma tentativa em que foi fornecida uma informação falsa e o participante foi solicitado a não oscilar. Nota: nos painéis a e c, a linha escura se refere ao movimento da sala e a linha mais clara às oscilações corporais dos participantes. 84 
FIGURA 30 - Exemplos de séries temporais de uma criança de 8 anos ao longo de uma tentativa mostrando o movimento da sala e a oscilação corporal na direção ântero-posterior (painel a), a fase relativa entre o movimento da sala e a oscilação corporal (painel b), e a análise espectral tanto do movimento da sala quanto da oscilação corporal (painel c), em uma tentativa em que foi fornecida uma informação correta e o participante foi solicitado oscilar junto com a sala. Nota: nos painéis a e c, a linha escura se refere ao movimento da sala e a linha mais clara às oscilações corporais dos participantes .85

FIGURA 31 - Exemplos de séries temporais de uma criança de 8 anos ao longo de uma tentativa mostrando o movimento da sala e a oscilação corporal na direção antero-posterior (painel a), a fase relativa entre o movimento da sala e a oscilação corporal (painel b), e a análise espectral tanto do movimento da sala quanto da oscilação corporal (painel c), em uma tentativa em que foi fornecida uma informação falsa e o participante foi solicitado a oscilar junto com a sala. Nota: nos painéis a e c, a linha escura se refere ao movimento da sala e a linha mais clara às oscilações corporais dos participantes .86 
FIGURA 32 - Médias e desvios padrão da amplitude média de oscilação, nas condições, sala com movimento e sala sem movimento, para os dois grupos etários com o fornecimento de informação verbal correta e falsa a respeito da movimentação da sala móvel. 88

FIGURA 33 - Médias e desvios padrão da frequiência média de oscilação, nas condições, sala com movimento e sala sem movimento, para os dois grupos etários com o fornecimento de informação verbal correta e falsa a respeito da movimentação da sala móvel.

FIGURA 34 - Médias e desvios padrão da coerência, nas duas condições em que a sala foi movimentada e a sala não foi movimentada, para os dois grupos etários com o fornecimento de informação correta (painel a) e com o fornecimento de informação falsa (painel b).

FIGURA 35 - Médias e desvios padrão do ganho, nas duas condições em que a sala foi movimentada e a sala não foi movimentada, para os dois grupos etários com o fornecimento de informação correta (painel a) e com o fornecimento de informação falsa (painel b).

FIGURA 36 - Médias e desvios padrão da fase relativa, nas duas condições em que a sala foi movimentada e a sala não foi movimentada, para os dois grupos etários com o fornecimento de informação correta (painel a) e com o fornecimento de informação falsa (painel b) ..94 FIGURA 37 - Médias e desvios padrão do desvio angular, nas duas condições em que a sala foi movimentada e a sala não foi movimentada, para os dois grupos etários com o fornecimento de informação correta (painel a) e com o fornecimento de informação falsa (painel b) ..94 


\section{LISTA DE QUADROS}

QUADRO 1 - Representação esquemática da movimentação da sala de forma discreta, informação fornecida sobre o movimento da sala e ação solicitada ao sujeito.

QUADRO 2 - Representação esquemática da movimentação da sala de forma contínua, informação fornecida sobre o movimento da sala e ação solicitada ao sujeito. .52 


\section{LISTA DE ANEXOS}

ANEXO I- Carta do comitê de ética 133

ANEXO II- Termo de consentimento 134 


\section{LISTA DE APÊ NDICES}

APÊNDICE I - Planilha contendo os dados do experimento 1 - Com e sem movimento discreto da sala móvel.........................................136

APÊNDICE II- Planilha contendo os dados do experimento 2 - Com e sem movimento contínuo da sala móvel. 142 


\title{
RESUM 0
}

\section{EFEITOS DA INFORMAÇÃO VERBAL NO ACOPLAMENTO ENTRE A INFORMAÇÃO VISUAL E OSCILAÇÃO CORPORAL}

\author{
Autor: ALAÉRCIO PEROTTI JUNIOR \\ Orientador: Prof. Dr. GO TANI \\ Co-orientador: Prof. Dr. JOSÉ ANGELO BARELA
}

O objetivo desse estudo foi verificar os efeitos da manipulação de informação, visual proveniente de uma sala móvel, verbal fornecida sobre o movimento da sala e sobre uma ação solicitada, na oscilação corporal em crianças e adultos. Participaram deste estudo 20 crianças e 20 adultos jovens, que permaneceram na posição ereta dentro de uma sala móvel. Os resultados revelaram que a dinâmica intrínseca do sistema, referente ao relacionamento entre informação visual e oscilação corporal, não é facilmente modificada pela informação comportamental. A manipulação dos tipos de informação verbal, sobre movimento da sala e solicitação de uma ação, altera o relacionamento entre informação visual e oscilação corporal na situação da sala móvel. Entretanto, esta alteração requer atuação contínua do participante e, ainda, a solicitação de uma ação é mais efetiva nesta alteração do que somente a informação sobre o que está ocorrendo. Finalmente, há mudanças desenvolvimentais em como estas 
diferentes informações são utilizadas para o controle de uma ação motora. Enquanto adultos jovens utilizam as informações fornecidas de forma mais adequada para a ação solicitada, crianças apresentam dificuldade em utilizar a informação fornecida ou realizar uma ação solicitada frente à dinâmica intrínseca do sistema.

Palavras chaves: controle postural, ciclo percepção-ação, informação comportamental, dinâmica intrínseca, ação motora. 


\begin{abstract}
EFFECTS OF THE VERBAL INFORMATION IN THE COUPLING BETWEEN THE VISUAL INFORMATION AND BODY OSCILLATTION
\end{abstract}

\author{
Author: ALAÉRCIO PEROTTI JÚNIOR \\ Adviser: Prof. Dr. GO TANI \\ Co-adviser: Prof Dr. JOSÉ ANGELO BARELA
}

The purpose of this study was to verify the effects of the manipulation of information, visual from a moving room, verbal informing about the movement of the room and about a requested action, in body sway of children and adults. Participated of this study 20 children and 20 young adults, who stood upright inside of a moving room. The results revealed that the system intrinsic dynamics, regarding the relationship between visual information and body sway, is not easily modified by behavioral information. The manipulation of the types of verbal information, about the moving room's movement and requesting a specific action, alters the relationship between visual information and body sway in the moving room situation. However, this change requires the participant's continuous attention and, moreover, requesting an action is more effective than only the information about what is happening. Finally, there are behavioral changes in how these different types of information are used for the control of a 
motor action. While young adults use the provided information in a more appropriate way to perform the requested action, children show difficulty in order to use the provided information or to accomplish an action requested due to the intrinsic dynamics of the system.

Keywords: postural control, perception-action cycle, behavioral information, intrinsic dynamics, motor action. 